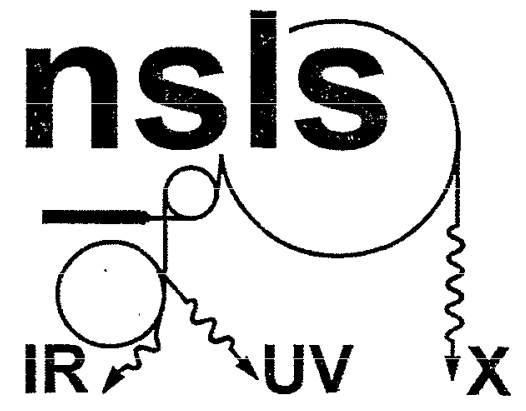

BNL-65983

INFORMAL REPORT

Design parameters of the high gain harmonic generation experiment using Cornell Undulator $A$ at the ATF

\title{
L.H. Yu
}

National Synchrotron Light Source

Brookhaven National Laboratory, P.O. Box 5000

Upton, NY 11973-5000

October 1998

\section{NATIONAL SYNCHROTRON LIGHT SOURCE}

\author{
BROOKHAVEN NATIONAL LABORATORY \\ BROOKHAVEN SCIENCE ASSOCIATES \\ Under Contract No. DE-AC02-98CH10886 with the \\ UNITED STATES DEPARTMENT OF ENERGY
}




\section{DISCLAIMER}

This report was prepared as an account of work sponsored by an agency of the United States Government. Neither the United States Government nor any agency thereof. nor any of their employees, nor any of their contractors, subcontractors, or their employees, makes any warranty, express or implied, or assumes any legal liability or responsibility for the accuracy, completeness, or usefulness of any information, apparatus, product, or process disclosed, or represents that its use would not infringe privately owned rights. Reference herein to any specific commercial product, process, or service by trade name, trademark, manufacturer, or otherwise, does not necessarily constitute or imply its endorsement, recommendation, or favoring by the United States Government or any agency, contractor or subcontractor thereof. The views and opinions of authors expressed herein do not necessarily state or reflect those of the United States Government or any agency, contractor or subcontractor thereof. 
BNL -65983

Informal Report

\title{
Design parameters of the high gain harmonic generation experment using Cornell Undulator A at the ATF
}

\author{
L.H. Yu \\ NSLS, Brookhaven National Laboratory, Upton, NY 11973, USA
}

\begin{abstract}
We present the design parameters of a high gain harmonic generation (HGHG) FEL experiment to be carried out at the accelerator test facility (ATF) at BNL, in collaboration with APS. This experiment is a proof-of-principle experiment for the DUV-FEL at BNL. In the $\mathrm{HGHG}$ experiment we plan to double the frequency of a $\mathrm{CO}_{2}$ seed laser by utilizing a $0.76 \mathrm{~m}$ long 9 period undulator (named the "Mini Undulator"), a $2 \mathrm{~m}$ long 60 period undulator (named the "Cornell Undulator A"), and a $0.3 \mathrm{~m}$ electromagnet chicane (the dispersive section). The first undulator will be used in conjunction with the $\mathrm{CO}_{2}$ seed laser to generate a ponderomotive force that will bunch the electron beam. The bunching will then be enhanced by the dispersion section. The second undulator, the Cornell Undulator A, tuned to the second harmonic of the seed laser will serve as the radiator. In the beginning of the radiator the bunched beam will produce coherent emission (characterized by a quadratic growth of the radiated power), then the radiation will be amplified exponentially. We plan to study the evolution of the various radiation growth mechanisms as well as the coherence of the doubled, exponentially amplified radiation.
\end{abstract}

\section{Introduction}

The seeded single pass FEL has many advantages over other FEL concepts. The output bandwidth is controlled by the input seed, limited only by the pulse length, and a bandwidth of $10^{-4}$ is possible. Similarly, frequency stability is also controlled by the seed; hence the electron beam energy stability influences only the output intensity fluctuations, relaxing the requirement on energy stability. Another obvious advantage is that the mirror loss and damage problems of FEL oscillators are eliminated. In addition, there is no need for a long train of micro-pulses. The electron beam can consist of single micro-pulses. High repetition rate can be provided by utilizing a superconducting linac. Thus, a seed beam makes it possible to achieve very good energy stability and high average power.

There are powerful, conventional high repetition-rate tunable lasers operating in the $\mathbb{R}$ and visible frequency bands which may be harmonic-multiplied into the VUV and used as seed lasers for the FEL amplifier. The interest in harmonic generation in FELs stems from the limitations of conventional laser harmonic generation techniques, such as low conversion efficiency, susceptibility to damage and limited tunability. 
The generation of harmonics by bunching an electron beam in a undulator, using a seed laser, is well known and verified experimentally [1]. The use of prebunching in FELs was studied analytically [2] and in a 3-D numerical simulation [3]. The extension of this technique to the exponential growth regime including undulator tapering has been proposed and studied in detail as the basis for the DUVFEL at BNL [4-6]. An order of magnitude improvement in the performance of this system can be obtained by further modifications of the technique [7]. However, the complete process of generating the harmonics by prebunching in the fundamental and amplification in a undulator tuned to the harmonic has not been demonstrated experimentally as yet. The generation of harmonics and subsequent exponential growth poses many interesting questions. It is our intention to pursue these questions experimentally in the experiment at the ATF.

In the proposed HGHG experiment, we will demonstrate the bunching of a $42 \mathrm{MeV}$ electron beam by a $\mathrm{CO}_{2}$ laser of nearly $0.7 \mathrm{MW}$ input power. We will study the coherent growth of the second harmonic at a wavelength of $5.3 \mu \mathrm{m}$, the exponential growth regime, and saturation regime. We would like to verify our theoretical models and to answer important questions such as the effect of electron beam parameters on the coherence of the FEL, the effect of undulator and alignment errors, and the higher harmonic contents of the FEL output near saturation.

We have selected the parameters of the harmonic generation experiment to match the electron beam parameters which have already been demonstrated experimentally at the ATF[8]. These include: a normalized rms emittance of $4 \pi \mathrm{mm} \mathrm{mrad}$ at a peak current of 110 Ampere, and the energy of $42 \mathrm{MeV}$; a $\mathrm{CO}_{2}$ oscillator with a pulse length of nearly 100 ns and a power of $0.7 \mathrm{MW}$; a solid-state optical chopper on the $\mathrm{CO}_{2}$ laser system which is synchronized to the electron beam. This chopper is capable of slicing a 10-100 ps long pulses. If necessary, these pulses can be amplified by a wide-band $\mathrm{CO}_{2}$ amplifier. In the first stage, we shall use the $0.7 \mathrm{Mw} 100 \mathrm{~ns}$ output of the oscillator output because its repetition rate, and because the chopper will lower the intensity. The amplifier repetition rate is lower than the oscillator, so only in the second stage when we need to increase the HGHG output power by increasing the $\mathrm{CO}_{2}$ power we will use the chopper and the amplifier.

\section{Parameters of the harmonic generation experiment}

The design and theory of the harmonic generation FEL has been described extensively in ref. [5]. Therefore we will confine this presentation to a brief description of the parameters of the experiment and the expected performance of the system.

Table 1 provides all the relevant parameters of the experiment: seed laser, electron beam, undulator, and expected FEL amplifier performance. The Rayleigh range, as well as the strength of the dispersive section have been optimized using a procedure, described in ref. [5]. 
Following the optimization of the FEL parameters, the complete simulation of the HGHG FEL has been done using a modified version of the computer code TDA [10].

A schematic diagram of the harmonic generation experiment is shown in fig. 1.

We will use a $\mathrm{CO}_{2}$ seed laser at a wavelength of $10.6 \mu \mathrm{m}$. The seed-laser pulse-length will be initially $100 \mathrm{~ns}$ at an input power of $0.7 \mathrm{MW}$. The electron-beam pulse-length at the ATF is assumed to be 4 ps FWHM.

Our system utilizes two undulator magnets separated by a dispersion section. The first undulator, the Mini-Undulator, is used to energy modulate the electron beam. At the end of the Mini-Undulator, the simulated energy modulation of particles versus the ponderomotive phase is given in figure 2. The period of this undulator is $8 \mathrm{~cm}$ and the peak axial magnetic field is $0.158 \mathrm{~T}$, making the $42 \mathrm{MeV}$ electron beam resonant with the $10.6 \mu \mathrm{m}$ seed radiation. This is followed the dispersion section with a magnetic field of 0.5 $\mathrm{T}$, in which the energy modulation is converted into a spatial bunching with a strong tsecond harmonic component at $5.3 \mu \mathrm{m}$. At the end of the dispersion section, the energy phase distribution is given in figure 3 , showing strong micro bunching.

When the coherently bunched beam enters the $2 \mathrm{~m}$ long radiator undulator magnet (the Cornell Undulator A, resonant at $5.3 \mu \mathrm{m}$ ), there is a rapid coherent generation of $5.3 \mu \mathrm{m}$ radiation within the first two gain lengths, ie., about 0.5 meter. The radiation has a characteristic quadratic dependence on the distance traversed in the undulator. Then there is a transition to exponential growth which continues until about $1.8 \mathrm{~m}$, where it reaches saturation, with output power of $45 \mathrm{Mw}$. At the end of the Cornell Undulator A, the energy phase distribution is plotted in figure 4 , showing saturation, and the $5 \mu \mathrm{m}$ structure instead of the $10 \mu \mathrm{m}$ structure in the figure 2 and 3.

The three distinct FEL processes occurring in the second wiggier (the quadratic superradiant growth, the exponential growth, and saturation) are shown clearly when the radiation power is plotted against the undulator length in figure 5.

We have looked into the sensitivity of the FEL performance on electron beam parameters, such as the emittance and energy spread. Changing the emittance, from 4 to $6 \times 10^{-6} \pi \mathrm{mrad}$ changes the power e-folding length of the second undulator from 0.26 to $0.29 \mathrm{~m}$. Changing the current from 110 ampere to 90 ampere again increases the gain length to $0.29 \mathrm{~m}$. Similarly, changing the FWHM energy spread from 0.1 to $0.2 \%$ changes the e-folding length from $0.26 \mathrm{~m}$ to $0.27 \mathrm{~m}$.

To study the tolerance on the undulator errors, we simulated the system by seeding the calculation with different sets of random undulator peak to peak errors. For simplicity, we calculate for the case seeded with 5 micron $\mathrm{CO}_{2}$ beam of peak power $10 \mathrm{kw}$. In figure 6 we plot the gain verses the rms displacement for each individual set of undulator errors. Each point in the plot represents one set of undulator error, and hence one particular 
trajectory. This plot shows that the output power is predominantly a function of the rms displacement. When the rms displacement is 100 micron, the gain drops from about 160 to about 120 . Hence we choose 100 micron as our tolerance on trajectory displacement.

\section{References}

[1] R. Prazeres, P. Guyot-Sionnest, J.M. Ortega, D. Jaroszynski, M. Billardon, M.E. Couprie, M. Velghe and Y. Petroff, Nucl. Instr. and Meth. A304 (1991) 72.

[2] I. Boscolo and V. Stagno, Nucl. Instr. and Meth. 188 (1982) 483.

[3] R. Bonifacio, L. de Salvo Souza, P. Pierini and E.T. Scharlemann, Nucl. Instr. and Meth. A296 (1990) 787.

[4] I. Ben-Zvi, L.F. Di Mauro, S. Krinsky, M.G. White and L.H. Yu, Nucl. Instr. and Meth. A304 (1991) 181.

[5] L.H. Yu, Phys. Rev. A44 (1991) 5178.

[6] I. Ben-Zvi, L.F. Di Mauro, S. Krinsky, M.G. White, L.H. Yu, K. Batchelor, A. Friedman, A.S. Fisher, H. Halama, G. Ingold, E. D. Johnson, S. Kramer, J.T. Rogers, L. Solomon, J. Wachtel and X. Zhang, Nucl. Instr. and Meth. A318 (1992) 201.

[7] I. Ben-Zvi, K.M. Yang and L.H. Yu, Nucl. Instr. and Meth. A318 (1992) 726.

[8] I. Ben-Zvi, The BNL accelerator test facility and experimental program, Proc. 1991 Particle Accelerator Conf., San Francisco, CA.

[9] L.H. Yu, S. Krinsky and RL. Gluckstern, Phys. Rev. Lett. 64 (25) (1990) 3011. [10] T.M. Tran and J.S. Wurtele, LRP 354/88, Ecole Polytechnique Federale de Lausanne, Suisse (1988).

[11] I. Ben-Zvi, R. Fernow, J. Gallardo, G. Ingold, W. Sampson and M. Woodle, Nucl. Instr. and Meth. A318 (1992) 781.

[12] E.T. Scharlemann, J. Appl. Phys. 58 (1985) 2154. 
Table I System parameters

Electron beam parameters

Energy $\gamma$

82

Current I

$110 \mathrm{~A}$

Micropulse length

Emittance (normalized, rms)

4 ps

$\mathrm{rms}$ beam size $\sigma_{\mathrm{x}}$ (matched beam)

$4 \times 10^{-6} \pi \mathrm{mrad}$

Local energy spread $\sigma_{\gamma} / \gamma$

$240 \mu \mathrm{m}$

$0.043 \%$

Seed laser parameters

Wavelength

Input power $P_{\text {in }}$

Pulse length, initial

Improved (sliced) pulse length

Rayleigh Range

$10.4 \mu \mathrm{m}$

$0.7 \mathrm{MW}$.

$100 \mathrm{~ns}$

$10-100$ ps

$0.76 \mathrm{~m}$

Modulator undulator:

Length

Period

$0.76 \mathrm{~m}$

Peak magnetic field

$8 \mathrm{~cm}$

$0.158 \mathrm{~T}$

Dispersive section:

Length

Magnetic field

$30 \mathrm{~cm}$

Dispersion $\left(\frac{d \psi}{d \gamma}\right)$

$0.5 \mathrm{~T}$

1.5

Radiator undulator (Cornell Undulator A):

$\begin{array}{ll}\text { Period } & 3.3 \mathrm{~cm} \\ \text { Length } & 2 \mathrm{~m} \\ \text { Exponential section magnetic field } & 0.47 \mathrm{~T} \\ \text { Betatron wavelength } & 3.75 \mathrm{~m}\end{array}$

FEL parameters:

Wavelength

$5.2 \mu \mathrm{m}$

Bessel factor $\mathrm{J}_{0}-\mathrm{J}_{1}$

0.857

Exponential section power e-folding length

$0.263 \mathrm{~m}$

Pierce parameter $\rho$

0.0089

Output power

$45 \mathrm{MW}$

Output energy for a 4 ps FWHM pulse

$\sim 0.2 \mathrm{~mJ}$ 


\section{Figures}

Figure 1: schematic diagram of the HGHG experiment

Figure 2: the energy phase diagram at the end of the Mini-Undulator

Figure 3: the energy phase diagram at the end of the dispersion section

Figure 4: the energy phase diagram at the end of the Cornell Undulator A

Figure 5: Radiation power versus distance in Cornell Undulator A

Figure 6: Gain for different simulation trajectories with different rms displacements, when the Cornell Undulator $\mathrm{A}$ is used as an amplifier. 


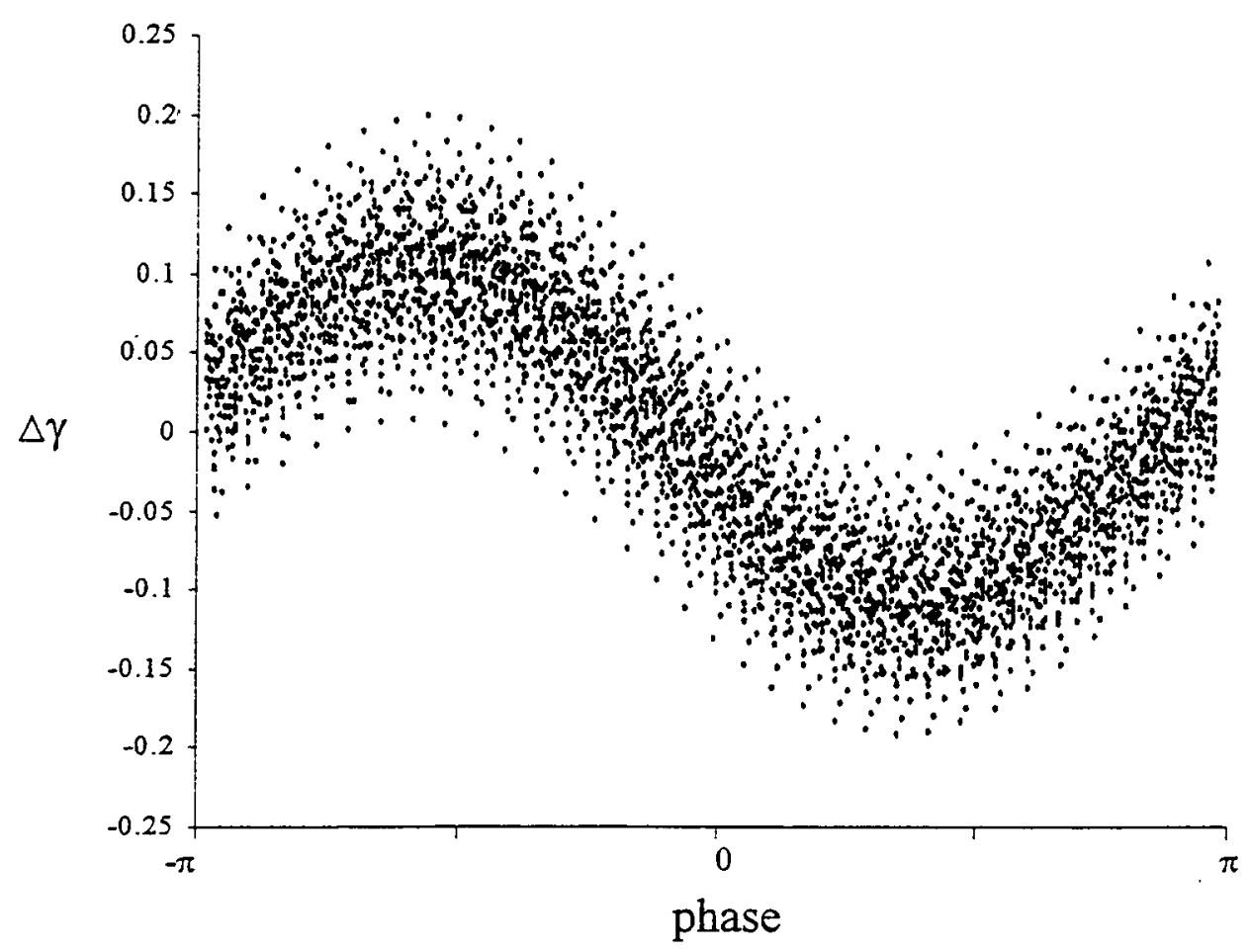

Figure 2 


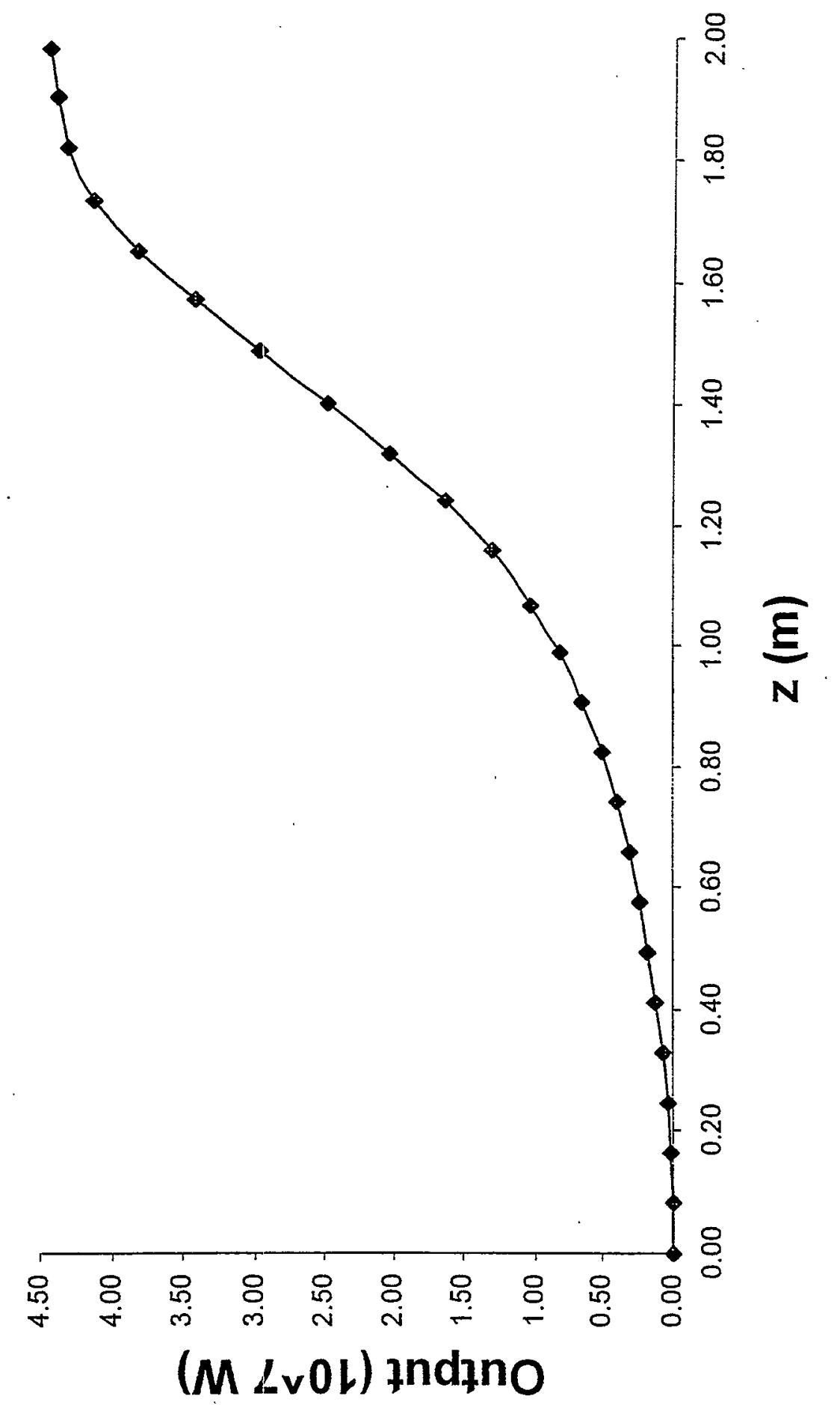

有 


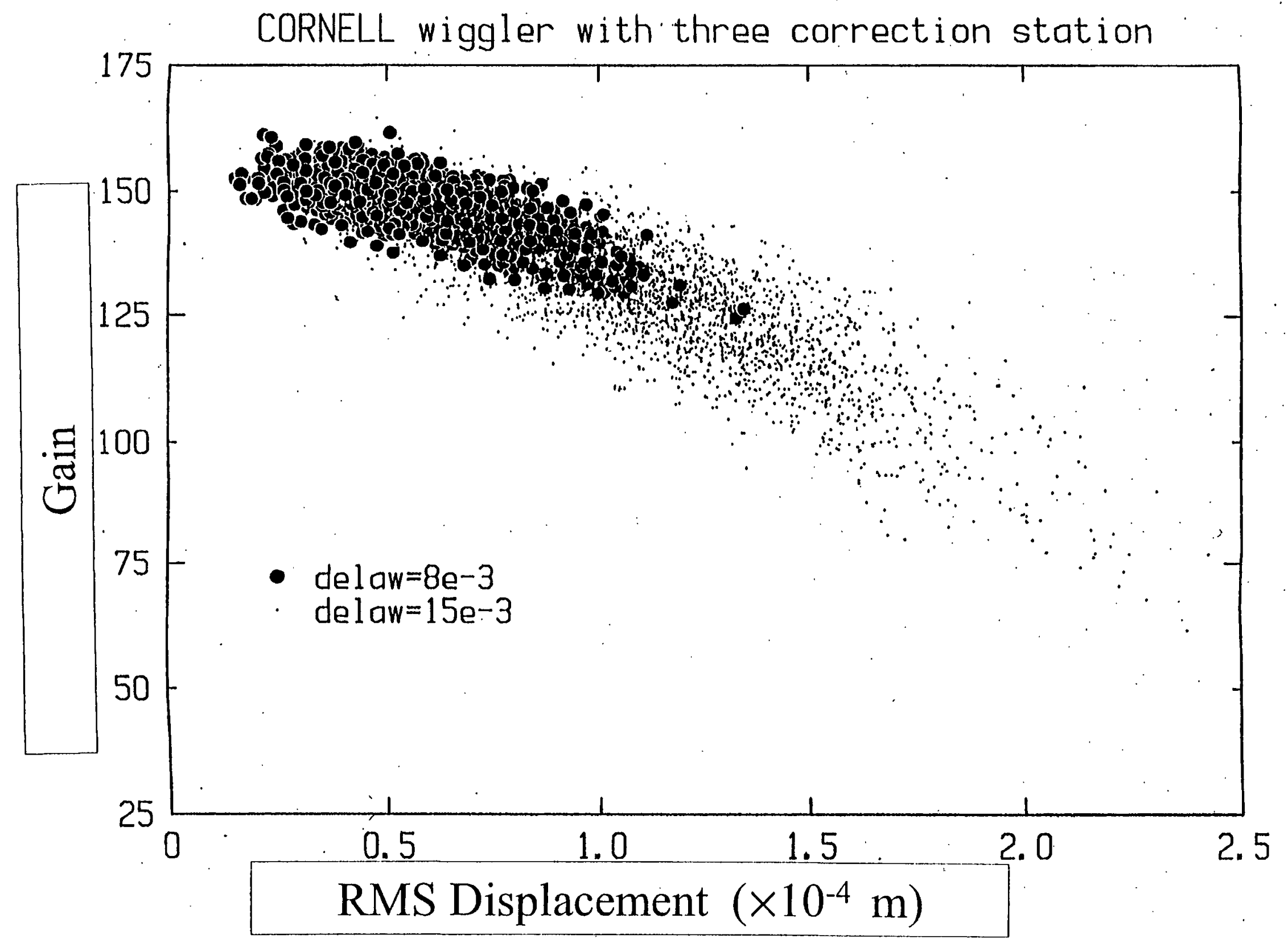

Figure 6 


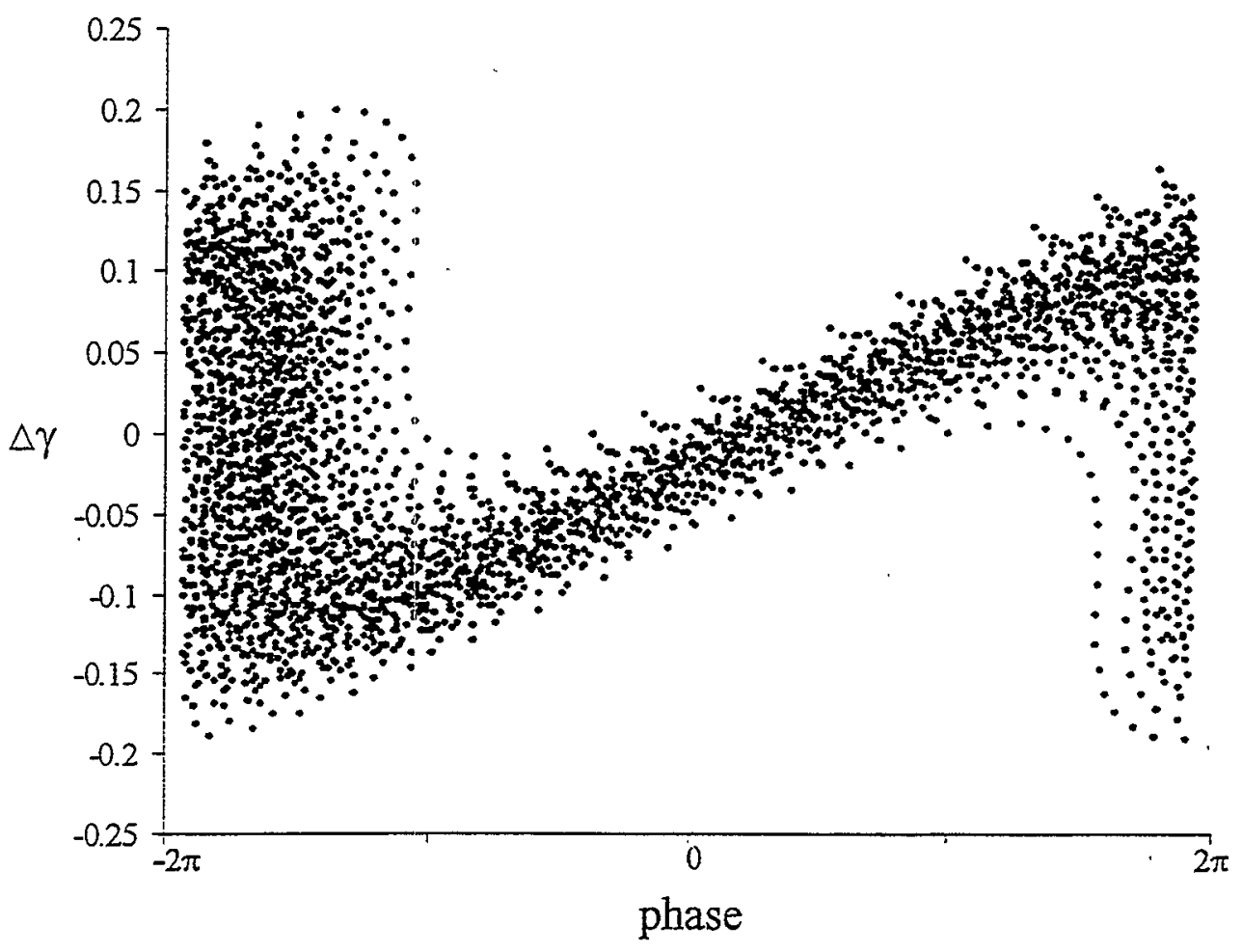

Figure 3 


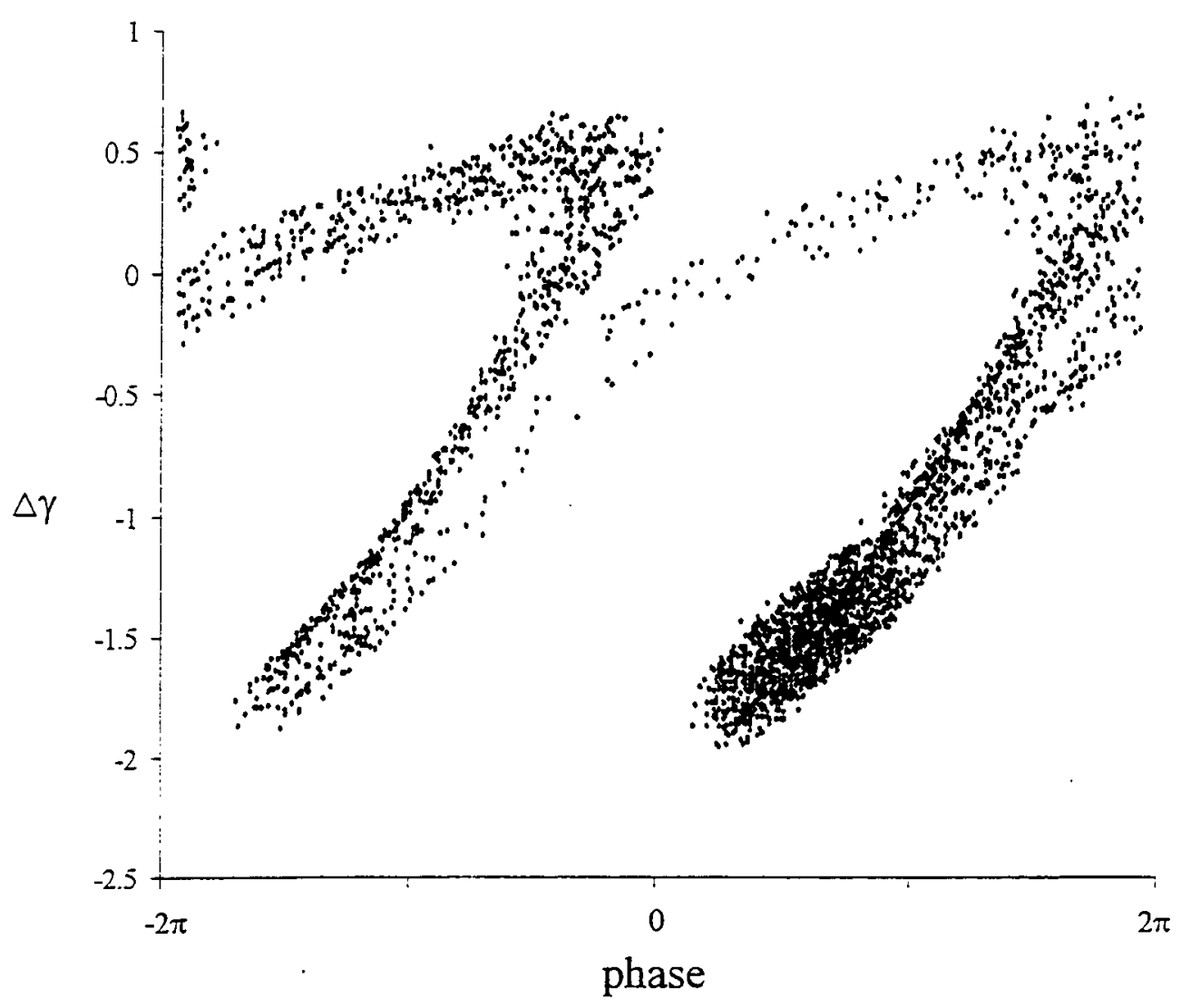

Figure 4 